\title{
Study on the relationship between age and the concentrations of heavy metal elements in human bone
}

\author{
Liang Chang ${ }^{1,2}$, Sheng Shen ${ }^{1}$, Zhe Zhang ${ }^{1,2}$, Xiaoxiao Song ${ }^{1,2}$, Qing Jiang ${ }^{1,2}$ \\ ${ }^{1}$ Department of Sports Medicine and Adult Reconstructive Surgery, Drum Tower Hospital affiliated to Medical School of Nanjing University, \\ Nanjing 210000, China; 'Joint Research Center for Bone and Joint Disease, Model Animal Research Center (MARC), Nanjing University, Nanjing \\ 210093, China \\ Contributions: (I) Conception and design: Q Jiang, S Shen, L Chang; (II) Administrative support: Q Jiang; (III) Provision of study materials or \\ patients: S Shen, Z Zhang, X Song; (IV) Collection and assembly of data: L Chang, Z Zhang, X Song; (V) Data analysis and interpretation: L Chang; \\ (VI) Manuscript writing: All authors; (VII) Final approval of manuscript: All authors. \\ Correspondence to: Professor Qing Jiang. The Center of Diagnosis and Treatment for Joint Disease, Drum Tower Hospital, Medical School, Nanjing \\ University, 321 Zhongshan Road, Nanjing 210008, China, Email: qingj@nju.edu.cn.
}

\begin{abstract}
Background: Excessive amounts of heavy metals such as cadmium, chromium, cobalt, lead, thallium, and manganese are extremely harmful to the human body. These elements can accumulate in bone and impact bone metabolism. In this study, we investigated the relationship between age and the concentrations of these elements in human bone and blood.

Methods: Bone and blood samples were obtained from both older and younger patients. The concentrations of the elements under investigation were measured by inductively coupled plasma mass spectrometry (ICP-MS), and the specific concentrations in the bone and blood were then calculated.

Results: The results showed that with increasing age, the concentrations of chromium, cobalt, and thallium in bone decreased significantly, while the concentration of cadmium in bone markedly increased. However, there was no clear correlation between age and the concentrations of these heavy metals in blood. Notably, there was a close correlation between the concentration of cobalt in bone and the presence of osteopenia.

Conclusions: Senescence of the human body is accompanied by the shifting of cobalt, chromium, and thallium from the bone to the outer- bone. However, the concentration of cadmium in bone increases with age. These changes are very likely to be related to the equilibrium of bone metabolism in senescent individuals. In addition, only cobalt was shown to be significantly related to osteopenia.
\end{abstract}

Keywords: Heavy metal elements; bone concentration; blood concentration; age; osteopenia

Submitted May 01, 2018. Accepted for publication Aug 03, 2018.

doi: 10.21037/atm.2018.08.09

View this article at: http://dx.doi.org/10.21037/atm.2018.08.09

\section{Introduction}

A heavy metal is a metal with a density greater than $4.5 \mathrm{~g} / \mathrm{cm}^{3}$. Depending on whether is the heavy metal is useful in the human body, these elements can be divided into essential heavy metal elements and non-essential heavy metal elements. Essential heavy metal elements include iron $(\mathrm{Fe})$, zinc $(\mathrm{Zn})$, copper $(\mathrm{Cu})$, manganese $(\mathrm{Mn})$, cobalt (Co), and tin (Sn), all of which are involved in important physiological processes such as the maintenance of water and electrolyte balance and the synthesis of enzymes and vitamins. Non-essential heavy metal elements mainly refer to cadmium (Cd), chromium $(\mathrm{Cr})$, lead $(\mathrm{Pb})$, thallium $(\mathrm{Tl})$, and arsenic (As), which are elements that have no utility in normal physiological functions. Excessive amounts of either essential heavy metals or non-essential heavy metals are extremely harmful to the human body (1). Among the heavy metals, $\mathrm{Cd}, \mathrm{Cr}, \mathrm{Co}, \mathrm{Pb}, \mathrm{Mn}$ and $\mathrm{Tl}$ are the most toxic. Compared with $\mathrm{Fe}, \mathrm{Zn}$ and $\mathrm{Cu}$, the above six heavy metals are scarcer and more toxic. A very small excess amount of 
these heavy metals can cause serious damage to tissues and organs.

There have been a large number of reports about the toxic effects of these heavy metal elements on the human body. For example, $\mathrm{Pb}$ exposure can lead to intellectual disability; Cd has profound nephrotoxic and osteotoxic activities; Co is teratogenic and closely related to interstitial lung disease; $\mathrm{Cr}$ can cause numbness of the limbs, mental disorders and carcinoma; and $\mathrm{Mn}$ and $\mathrm{Tl}$ severely affect cognitive function through damage to the central nervous system (2-7). Heavy metal elements are mainly ingested in food or absorbed through environmental exposure and then are distributed to tissues and organs throughout the whole body via the digestive tract and blood circulation. Since large amounts of heavy metals, which have long half-lives, are distributed in the skeleton, the skeleton is considered a long-term storage site for heavy metal elements.

With increased age, increasing numbers of senescent cells accumulate in tissues and organs. These senescent cells can secrete immune-stimulating factors, cause inflammatory reactions, and decrease the viability of surrounding tissues and cells (8). Therefore, along with the process of senescence, human bones will be more sensitive to heavy metals, which is likely to affect the distribution of heavy metal elements in the skeleton. To the best of our knowledge, there have been no reports about the relationship between age and the concentrations of $\mathrm{Cd}, \mathrm{Cr}, \mathrm{Co}, \mathrm{Pb}, \mathrm{Mn}$ and $\mathrm{Tl}$ in bone.

In our study, we used inductively coupled plasma mass spectrometry (ICP-MS) to determine the concentrations of heavy metal elements in the bones and blood of both younger and older individuals. A nonparametric test was used for comparative analysis. Since many heavy metal elements have been documented as influencing bone metabolism directly or indirectly, we also investigated the correlations between the concentrations of heavy metals and bone metabolism.

\section{Methods}

\section{Sampling}

Cancellous bone from the greater trochanter was obtained during total hip arthroplasty in the older adults (age $>45$, $\mathrm{n}=16$ ), and cancellous bone from the tibia was obtained during anterior cruciate ligament reconstruction surgery in the younger individuals (age $<35, \mathrm{n}=7$ ). All the bone samples were weighed (wet weight) and the weights were recorded. For every patient, $2-3 \mathrm{~mL}$ of venous blood was sampled three days after surgery, and the bone mineral density (BMD) was measured on the forearm by dual energy X-ray absorptiometry (DEXA) to categorize patients in the group of older adults as belonging to the normal group $(n=9)$ or the osteopenia group $(n=7)$. Osteopenia is a term to define BMD that is below the normal level but not as low as the upper limit defining osteoporosis. According to the World Health Organization, osteopenia is defined as a bone densitometry $\mathrm{T}$ score between -1 and -2.5 , while osteoporosis is defined as a bone densitometry $\mathrm{T}$ score $\leq-2.5$. In our study, the inclusion criteria for the osteopenia group was defined as a $\mathrm{T}$ score $<-1$, including both osteopenia and osteoporosis patients. Furthermore, the exclusion criteria were a history of chronic diseases, tumors, autoimmune diseases, and viral chronic infections (hepatitis $\mathrm{B}$ virus, hepatitis $\mathrm{C}$ virus, human immunodeficiency virus); previous orthopedic surgical implants; alcohol abuse; and exposure to toxic heavy metals. All patients provided informed consent, and this study was approved by the ethics committee of Nanjing Drum Tower Hospital, which is affiliated with the Medical School of Nanjing University (No. 2009022). The general characteristics of the entire study group are shown in Table 1 .

\section{Preparation of test diluent}

Bone samples were dried in a Biosafer-10C Lyophilizer (Nanjing Safer Biotech Co., Ltd., China) at -40 to $-50{ }^{\circ} \mathrm{C}$ for 2 days until the weight was constant; then, the samples were reweighed (dry weight) and the rate of weight loss was calculated. For the preparation of the test liquid, first, the bone samples were placed in a $25-\mathrm{mL}$ angled-bottomed flask; then, $2 \mathrm{~mL}$ of concentrated nitric acid (A0300005, GB-626 Analytical Reagent, Beijing Chemical Factory, Beijing, China) was added, and the bone fragments were left to soak overnight. Next, $6 \mathrm{~mL}$ of $30 \%$ hydrogen peroxide (A1029005, HG3-1082-77 Analytical Reagent, Beijing Chemical Factory, Beijing, China) was added, and the mixture was digested at $90-100{ }^{\circ} \mathrm{C}$ for 24 hours with a magnetic heated stirrer (KMS-181E, Shanghai Keezo Technology Co., Ltd., Shanghai, China). The transparent digestion solutions were diluted with ultrapure water to $20 \mathrm{~mL}$ and stored at $4{ }^{\circ} \mathrm{C}$. For each blood sample, a $1 \mathrm{~mL}$ blood sample was transferred to a $50-\mathrm{mL}$ angled-bottomed flask, and then $1 \mathrm{~mL}$ of concentrated nitric acid was added into the flask and left overnight. The next day, $10 \mathrm{~mL}$ of $30 \%$ hydrogen peroxide was added, and the mixture was digested at $90-100{ }^{\circ} \mathrm{C}$ for 24 hours. Then the solutions were 
Table 1 General characteristics

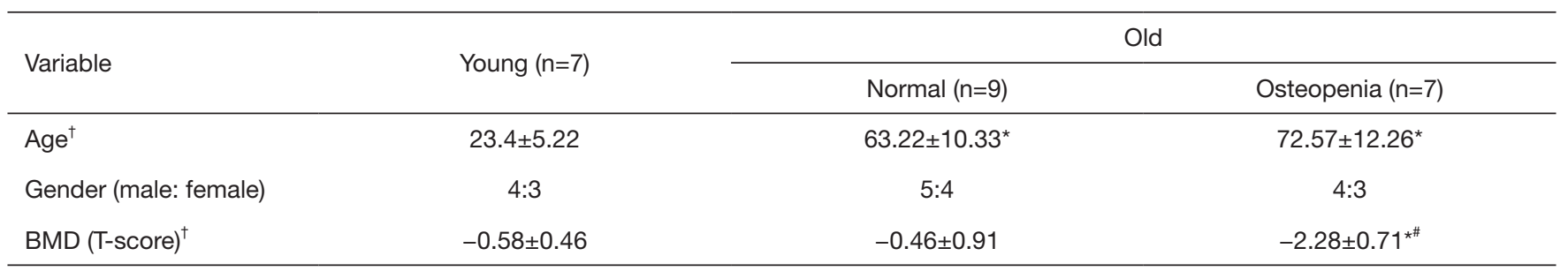

${ }^{\dagger}$, data are described as the mean $\pm \mathrm{SD}$; ${ }^{*}$, both the normal group and the osteopenia group were compared with the young group, $\mathrm{P}<0.05$;

\#, the osteopenia group was compared with the normal group, $\mathrm{P}<0.05$. BMD, bone mineral density; SD, standard deviation.

Table 2 Dry-wet ratio of bone samples ${ }^{\dagger}$

\begin{tabular}{lccc}
\hline \multirow{2}{*}{ Variable } & Young $(\mathrm{n}=7)$ & & Old \\
\cline { 3 - 4 } & & Normal $(\mathrm{n}=9)$ & Osteopenia $(\mathrm{n}=7)$ \\
\hline Wet weight $(\mathrm{g})$ & $0.331 \pm 0.189$ & $0.961 \pm 0.335$ & $0.895 \pm 0.369$ \\
Dry weight $(\mathrm{g})$ & $0.213 \pm 0.132$ & $0.551 \pm 0.207$ & $0.403 \pm 0.209$ \\
Dry-wet ratio $(\%)$ & $65.58 \pm 14.83$ & $57.52 \pm 7.46$ & $43.03 \pm 8.3^{* \#}$ \\
\hline
\end{tabular}

${ }^{\dagger}$, data are described as the mean $\pm \mathrm{SD}$; ${ }^{*}$, both the normal group and the osteopenia group were compared with the young group, $\mathrm{P}<0.05$;

\#, the dry-wet ratio of osteopenia group was compared with the normal group, $\mathrm{P}<0.05$.

diluted to $20 \mathrm{~mL}$ and stored at $4{ }^{\circ} \mathrm{C}$.

\section{Measurements}

ICP-MS is the most sensitive and commercial instrument for the determination of the concentrations of a wide range of metals. The advantages of ICP-MS as an elemental detector include low detection limits $(\mathrm{pg} / \mathrm{mL}$ level for most elements), low matrix effects, wide dynamic ranges, and high spectral resolution for elements. In our study, we used ICP-MS to determine the concentrations of the toxic heavy metal elements in the bone and blood diluent, and then calculated the corresponding concentrations of the heavy metals in the bone $(\mathrm{ng} / \mathrm{g})$ and blood $(\mathrm{ng} / \mathrm{mL})$. An Agilent 7700x ICP-MS (Agilent Technologies, California, USA) was provided by Nanjing FocuMS Technology Co. Ltd., and it has a detection accuracy of ppb. For specific operations, the test diluent was first diluted with $18.2 \mathrm{M} \Omega$ of ultrapure water to a total solid soluble content of less than $1.2 \mathrm{wt} \%$; then, the diluent was loaded into the Agilent 7700x ICPMS in an atomized form for the determination of the concentrations of the trace elements.

\section{Statistical analysis}

All data are described as the mean \pm standard deviation (SD) and were analyzed by SPSS Statistics 23.0 software (SPSS Inc., Chicago, IL, USA) using the Mann-Whitney U-test (nonparametric test). The results were compared among the young group, the normal group, and the osteopenia group. A $\mathrm{P}$ value $<0.05$ was considered statistically significant. Pearson's correlation analysis was carried out to determine the relationship between the concentrations of heavy metals in the bone and osteopenia.

\section{Results}

\section{Dry-wet ratio of bone samples}

Since the dry-wet ratio of bone can reflect the level of BMD to a certain extent, we calculated the dry-wet ratios of the bone samples to ascertain whether the BMD data from the forearm accurately reflected the BMD of the bone samples. The results are shown in Table 2, and they demonstrated that compared with both the normal group and the young group, the osteopenia group had a significantly lower drywet ratio of the bone, suggesting that the basis for grouping in our study was reliable.

\section{Analysis of the concentrations of heavy metal elements in blood}

The concentrations of the toxic heavy metal elements $\mathrm{Cd}$, 
Table 3 The concentrations of heavy metal elements in blood $(\mathrm{ng} / \mathrm{mL})^{\dagger}$

\begin{tabular}{lccr}
\hline Element & Young $(n=7)$ & Normal $(n=9)$ & Osteopenia $(n=7)$ \\
\hline Cd & $5.23 \pm 0.89$ & $6.8 \pm 4.86$ & $5.66 \pm 2.15$ \\
$\mathrm{Cr}$ & $474.92 \pm 242.68$ & $437.82 \pm 192.07$ & $322.67 \pm 33.61$ \\
$\mathrm{Co}$ & $4.48 \pm 1.58$ & $4.2 \pm 1.56$ & $4.03 \pm 1.68$ \\
$\mathrm{~Pb}$ & $207.97 \pm 94.75$ & $176.87 \pm 86.4$ & $138.89 \pm 64.66$ \\
$\mathrm{Mn}$ & $114.38 \pm 62.4$ & $118.33 \pm 70.8$ & $153.39 \pm 75.48$ \\
$\mathrm{Tl}$ & $72.79 \pm 54.69$ & $65.13 \pm 25.58$ & $60.08 \pm 10.2$ \\
\hline
\end{tabular}

${ }^{\dagger}$, data are described as the mean \pm SD. SD, standard deviation.

Table 4 The concentrations of heavy metal elements in bone (ng/g) ${ }^{\dagger}$

\begin{tabular}{lccc}
\hline Element & Young $(\mathrm{n}=7)$ & Normal $(\mathrm{n}=9)$ & Osteopenia $(\mathrm{n}=7)$ \\
\hline $\mathrm{Cd}$ & $27.65 \pm 19.03$ & $80.7 \pm 41.48^{*}$ & $112.02 \pm 92.09^{\star}$ \\
$\mathrm{Cr}$ & $5284.80 \pm 1996.68$ & $1568.25 \pm 969.43^{*}$ & $1572.49 \pm 938.37^{*}$ \\
$\mathrm{Co}$ & $57.63 \pm 32.23$ & $17.67 \pm 9.51^{*}$ & $38.42 \pm 20.84^{*}$ \\
$\mathrm{~Pb}$ & $1697.18 \pm 664.03$ & $1626.61 \pm 842.23$ & $1818.34 \pm 928.27$ \\
$\mathrm{Mn}$ & $1536.43 \pm 691.97$ & $704.24 \pm 221.13$ & $1685.0 \pm 1252.30$ \\
$\mathrm{Tl}$ & $2810.35 \pm 330.75$ & $1903.68 \pm 565.5^{*}$ & $2251.45 \pm 977.37^{*}$ \\
\hline
\end{tabular}

${ }^{\dagger}$, data are described as the mean $\pm \mathrm{SD}$; ${ }^{*}$, both the normal group and the osteopenia group were compared with the young group, $\mathrm{P}<0.05$;

\#, the osteopenia group was compared with the normal group, $\mathrm{P}<0.05$. SD, standard deviation.

Table 5 Pearson's correlation analysis between osteopenia and concentrations of heavy metal elements in bone

\begin{tabular}{lc}
\hline Element & T Score $(n=16) r(P)^{\#}$ \\
\hline $\mathrm{Co}$ & $-0.6867(0.0136)^{\star}$ \\
$\mathrm{Cd}$ & $-0.2712(0.3939)$ \\
$\mathrm{Cr}$ & $0.4974(0.0999)$ \\
$\mathrm{TI}$ & $0.2077(0.5171)$ \\
$\mathrm{Pb}$ & $0.2789(0.3801)$ \\
$\mathrm{Mn}$ & $-0.1524(0.6362)$ \\
\hline
\end{tabular}

\#, correlation coefficient ( $\mathrm{P}$ value); ${ }^{*}, \mathrm{P}<0.05$.

$\mathrm{Cr}, \mathrm{Co}, \mathrm{Pb}, \mathrm{Mn}$, and $\mathrm{Tl}$ in the blood samples are shown in Table 3. Our results demonstrated no significant differences, suggesting that there is no correlation between age, osteopenia and the blood concentrations of heavy metals, which means that the intake of abovementioned six heavy metals from diet and environment maintains at a steady level in all the samples.

\section{Analysis of the concentrations of heavy metal elements in bone}

The concentrations of the toxic heavy metal elements $\mathrm{Cd}$, $\mathrm{Cr}, \mathrm{Co}, \mathrm{Pb}, \mathrm{Mn}$, and $\mathrm{Tl}$ in the bone samples are shown in Table 4. Our results showed that the bone concentrations of $\mathrm{Cr}, \mathrm{Co}$, and $\mathrm{Tl}$ in the normal group were significantly lower than those in the young group, which indicates that the bone concentrations of $\mathrm{Cr}, \mathrm{Co}$, and $\mathrm{Tl}$ decrease with increasing age. Notably, the bone concentration of $\mathrm{Cd}$ increased significantly with age. In addition, the bone concentration of Co was significantly different between the normal group and the osteopenia group, suggesting that a higher concentration of Co in the bone was closely related to osteopenia in older adults. Pearson's correlation analysis was also performed, and that analysis verified the relationship between Co concentration in human bone and osteopenia (Table 5).

\section{Discussion}

In recent years, there have been many reports about the 
concentrations of heavy metals in bone. For example, Scimeca et al. proved that $\mathrm{Cd}, \mathrm{Cr}, \mathrm{Co}$, and $\mathrm{Pb}$ accumulated in the bones of osteoporosis and osteoarthritis patients (9), which emphasized the role these elements played in the pathogenesis of osteoporosis and osteoarthritis. Suwazono et al. and Akbal et al. demonstrated that low-dose Cd exposure and $\mathrm{Pb}$ exposure could induce osteoporosis $(10,11)$. Therefore, to explore the relationship between age and the concentrations of heavy metals in bone more accurately, osteoporosis and osteopenia patients should be considered independently.

The reason that studies in this field are rare is mainly because of the difficulty of obtaining bone samples from young individuals. Due to limited conditions, in our study, the bone samples for the younger group were obtained from the tibial tunnels of patients undergoing cruciate ligament reconstruction surgery. However, the bone samples for the older group were taken from the femoral greater trochanter of patients undergoing total hip arthroplasty. The reasons for our sampling choices are as follows: (I) the femoral greater trochanter and the proximal part of the tibia have similar biomechanical characteristics and both are nonstress concentration areas (12-15). According to Wolff's laws, the structure of the skeleton changes with the stress load it receives; thus, the two parts should have similar bone structures; (II) previous reports have shown that there is no significant difference in the concentrations of trace elements, including $\mathrm{Cd}, \mathrm{Cr}, \mathrm{Zn}, \mathrm{Fe}, \mathrm{Ca}$, and many other metal elements, between the femur and the tibia in humans, chickens, and rabbits (16-19). Therefore, we believe that the cancellous bone samples we obtained from the femur and the tibia have consistent structures and can be used for side-by-side comparisons.

Regarding the concentrations of heavy metals in blood, our results showed no correlation between the concentrations, age and osteopenia, which is consistent with Lim et al.'s research (20). Since the concentrations in the blood mainly reflect the intake levels of heavy metals from the external environment and diet, our data suggested that the history of exposure to heavy metals was consistent in all patients. In addition, we made a rough estimate of the distribution of the abovementioned six metals in blood and bone based on our test results. In a $60-\mathrm{kg}$ adult, the systemic skeletal weight accounts for $10 \%$ to $12 \%$ of the total body weight, that is, 6-7.2 $\mathrm{kg}$ (21). Systemic blood accounts for $7 \%$ to $8 \%$ of the total body weight, that is, $4.2-4.8 \mathrm{~kg}$, or approximately 4,000-4,600 mL. The concentrations of the six heavy metals investigated in this study were higher in the bone than in the blood, confirming the significance of the human skeleton for the retention of heavy metal elements.

Since many heavy metals have been shown to have close relationships with bone metabolism, our study attempted to analyze the physiological significance of the changes in the distribution of the abovementioned six heavy metals that occurred in the skeleton by determining the association between these heavy metals and bone metabolism. It is known that the concentrations of $\mathrm{Cr}$, $\mathrm{Co}$, and $\mathrm{Tl}$ in the bone decrease with age. In addition, the concentration of Co in the bone in the osteopenia group was significantly higher than that in the normal group, although it was still lower than that in the young group. According to previous reports, Co has a cytotoxic effect on osteoblasts and inhibits osteoblasts from secreting and expressing type I collagen. Similarly, Cr also inhibits the activity of osteoblasts (22), and Tl exerts a strong toxic effect on cells by interfering with DNA synthesis, which can cause skeletal and cartilage dysplasia $(23,24)$. Consequently, Cr, $\mathrm{Co}$, and $\mathrm{Tl}$ are potentially harmful factors affecting bone formation. Therefore, it is plausible to assume that along with the process of senescence, the transference of $\mathrm{Cr}, \mathrm{Co}$, and $\mathrm{Tl}$ out of the bone may contribute to maintaining the equilibrium of bone metabolism. Notably, the obstruction of this transfer process may induce osteopenia, as we observed for Co.

Regarding Cd, we observed that it accumulates in bone with increasing age. $\mathrm{Cd}$ is an element that is extremely nephrotoxic and osteotoxic. Its nephrotoxicity mainly manifests as damage to the renal tubules and glomeruli, which affects $\mathrm{Ca}$ and $\mathrm{P}$ metabolism, while its osteotoxicity manifests as a significant acceleration of bone loss, leading to osteoporosis. The classic theory presumes that the osteotoxicity of $\mathrm{Cd}$ is a secondary effect of kidney damage, resulting from the imbalance of $\mathrm{Ca}$ and $\mathrm{P}$ metabolism caused by renal injury (2). Therefore, the accumulation of $\mathrm{Cd}$ in the skeleton with increasing age may actually maintain the stability of bone metabolism by protecting renal function.

$\mathrm{Mn}$ is harmful to the central nervous system. Overexposure to Mn can lead to progressive, permanent, neurodegenerative damage, resulting in cognitive impairment $(2,7)$. Studies have shown that the lower the concentration of $\mathrm{Mn}$ in the bones of patients with osteoporosis, the greater the risk is of cognitive impairment such as Alzheimer's disease and Parkinson's disease (25). The abovementioned studies suggest that the level of $\mathrm{Mn}$ in bone is closely related to cognitive impairment in the 
brain. In addition, $\mathrm{Mn}$ can participate in the bone formation process, and studies have shown that Mn can significantly promote bone formation and increase BMD (26). Furthermore, Mn deficiency inhibits bone development by significantly reducing the mRNA expression ratio of OPG/RNAKL (27). In our study, we did not observe any relationship between age and the concentration of $\mathrm{Mn}$ in the bone, suggesting that the concentration of $\mathrm{Mn}$ in the bone is generally maintained at a stable level from adulthood, thereby not only maintaining the balance of bone metabolism but also preventing $\mathrm{Mn}$ from injuring the central nervous system.

The effect of $\mathrm{Pb}$ on bone metabolism is complex, and it can impair both osteoblasts and osteoclasts, although osteoclasts may be more sensitive than osteoblasts (28-30). Since the human skeleton is the most important storage site for $\mathrm{Pb}$, the concentration of $\mathrm{Pb}$ in the bone has been widely used in the clinical assessment of the $\mathrm{Pb}$ load in the body. No correlation between age and the concentration of $\mathrm{Pb}$ in the bone was observed in our study, suggesting that the concentration of $\mathrm{Pb}$ in the bone is also maintained at a stable level from adulthood, which may have considerable significance for bone metabolism and human health.

According to Scimeca's research, $\mathrm{Pb}, \mathrm{Cd}, \mathrm{Cr}$, and $\mathrm{Co}$ accumulate in the bones of osteoporotic patients. However, in our study, only the concentration of Co in the bone was observed to be highly correlated with osteopenia. This difference may have resulted from the fact that we had a small sample size. Further studies still need to be done to elucidate this observation. It is noteworthy that the increase in the concentration of Co in osteoporotic bone was not due to Co accumulation but rather to an obstruction of the process of Co reduction. There were several limitations in this study, such as the insufficient sample size and the difference in the sampling location between age groups. Although previous articles have demonstrated that there are no significant differences in the concentrations of heavy metals in the femur and the tibia, the difference in the sampling location is still a potential influencing factor.

In conclusion, we observed that in comparison to blood, the skeleton retains greater concentrations of heavy metals, and its storage capacity may change with age. According to our hypothesis, this change is associated with the balance of bone metabolism. Thus, those heavy metals that inhibit bone formation, such as $\mathrm{Cr}$, Co, and $\mathrm{Tl}$, gradually shift from the bone to the outer-bone with increasing age. Heavy metals that do not exert negative effects on bone metabolism, such as $\mathrm{Mn}$ and $\mathrm{Pb}$, have concentrations in the bone that are maintained at a stable level from adulthood. For Cd, its osteotoxicity is mainly secondary to renal injury; thus, as organs become senescent with age, the kidney becomes more sensitive to $\mathrm{Cd}$. The body tends to reduce renal Cd levels, which may indirectly lead to an increase in the concentration of $\mathrm{Cd}$ in the bone. These changes in the distribution of toxic heavy metals with age are very likely to be related to the high toxicity risk of these elements for older adults. However, the underlying mechanisms or metabolic pathways of this transference are still unknown, and further studies need to be conducted to elucidate the possible mechanisms. Notably, Co was observed to be closely related to osteopenia.

\section{Acknowledgments}

Funding: This study was supported by the Projects of International Cooperation and Exchanges NSFC (81420108021), National Key Technology Support Program (2015BAI08B02), Excellent Young Scholars NSFC (81622033), and the Project of Invigorating Health Care through Science, Technology and Education.

\section{Footnote}

Conflicts of Interest: The authors have no conflicts of interest to declare.

Ethical Statement: All patients provided informed consent, and this study was approved by the ethics committee of Nanjing Drum Tower Hospital, which is affiliated with the Medical School of Nanjing University (No. 2009022).

\section{References}

1. Jaishankar M, Tseten T, Anbalagan N, et al. Toxicity, mechanism and health effects of some heavy metals. Interdiscip Toxicol 2014;7:60-72.

2. Dermience M, Lognay G, Mathieu F, et al. Effects of thirty elements on bone metabolism. J Trace Elem Med Biol 2015;32:86-106.

3. Chmielowska-Bąk J, Izbiańska K, Deckert J. The toxic Doppelganger on the ionic and molecular mimicry of cadmium. Acta Biochimica Polonica 2013;60:369-74.

4. Junaid M, Hashmi MZ, Malik RN, et al. Toxicity and oxidative stress induced by chromium in workers exposed from different occupational settings around the globe: A review. Environ Sci Pollut Res Int 
2016;23:20151-67.

5. Paustenbach DJ, Tvermoes BE, Unice KM, et al. A review of the health hazards posed by cobalt. Crit Rev Toxicol 2013;43:316-62.

6. Cvjetko P, Cvjetko I, Pavlica M. Thallium toxicity in humans. Arh Hig Rada Toksikol 2010;61:111-9.

7. Peres TV, Schettinger MR, Chen P, et al. "Manganeseinduced neurotoxicity: a review of its behavioral consequences and neuroprotective strategies". BMC Pharmacol Toxicol 2016;17:57.

8. Wang P, Chen LP, Li QQ, et al. Cellular Senescence and Senescent Cell. Prog Biochem Biophys 2012;39:257-63.

9. Scimeca M, Feola M, Romano L, et al. Heavy metals accumulation affects bone microarchitecture in osteoporotic patients. Environ Toxicol 2017;32:1333-42.

10. Suwazono Y, Sand S, Vahter M, et al. Benchmark dose for cadmium-induced osteoporosis in women. Toxicol Lett 2010;197:123-7.

11. Akbal A, Tutkun E, Yilmaz H. Lead exposure is a risk for worsening bone mineral density in middle-aged male workers. Aging Male 2014;17:189-93.

12. Seo JW, Kang DW, Kim JY, et al. Finite element analysis of the femur during stance phase of gait based on musculoskeletal model simulation. Biomed Mater Eng 2014;24:2485-93.

13. Zhang J, Deng S, Zheng H, et al. The Establishment and Static Analysis of the Finite Element Model of the Human Femur. J B Inst Petrochem Technol 2012;20.

14. Rao C, Fitzpatrick CK, Rullkoetter PJ, et al. A statistical finite element model of the knee accounting for shape and alignment variability. Med Eng Phys 2013;35:1450-6.

15. Tang Y, Wei S, Cheng G, et al. A comparison between two finite element analysis methods of human tibia via different modeling patterns. Chin Orthop J Clin Basic Res 2016;8.

16. Hakki SS, Dundar N, Kayis SA, et al. Boron enhances strength and alters mineral composition of bone in rabbits fed a high energy diet. J Trace Elem Med Biol 2013;27:148-53.

17. Brodziak-Dopierała B, Roczniak W, Jakóbik-Kolon A, et al. Correlations between iron content in knee joint tissues and chosen indices of peripheral blood morphology. Adv Clin Exp Med 2017;26:1077-83.

18. Saeed AA, Sandhu MA, Khilji MS, et al. Effects of dietary chromium supplementation on muscle and bone mineral interaction in broiler chicken. J Trace Elem Med Biol 2017;42:25-9.

19. Roczniak W, Brodziak-Dopierala B, Cipora E, et al.
Factors that Affect the Content of Cadmium, Nickel, Copper and Zinc in Tissues of the Knee Joint. Biol Trace Elem Res 2017;178:201-9.

20. Lim HS, Lee HH, Kim TH, et al. Relationship between Heavy Metal Exposure and Bone Mineral Density in Korean Adult. J Bone Metab 2016;23:223-31.

21. Chen R, Wang Z, Ou Yang BY, et al. Reference values of skeleton mass for normal Chinese adults. Zhonghua fang she yi xue yu fang hu za zhi 1995;15.

22. Zijlstra WP, Bulstra SK, van Raay JJ, et al. Cobalt and chromium ions reduce human osteoblast-like cell activity in vitro, reduce the OPG to RANKL ratio, and induce oxidative stress. J Orthop Res 2012;30:740-7.

23. Rodríguez-Mercado JJ, Altamirano-Lozano MA. Genetic toxicology of thallium: a review. Drug Chem Toxicol 2013;36:369-83.

24. Léonard A, Gerber G. Mutagenicity, carcinogenicity and teratogenicity of thallium compounds. Mutat Res 1997;387:47-53.

25. Landete-Castillejos T, Molina-Quilez I, Estevez JA, et al. Alternative hypothesis for the origin of osteoporosis: the role of Mn. Front Biosci (Elite Ed) 2012;4:1385-90.

26. Bae YJ, Kim MH. Manganese supplementation improves mineral density of the spine and femur and serum osteocalcin in rats. Biol Trace Elem Res 2008;124:28-34.

27. Liu R, Jin C, Wang Z, et al. Effects of manganese deficiency on the microstructure of proximal tibia and OPG/RANKL gene expression in chicks. Vet Res Commun 2015;39:31-7.

28. Beier EE, Holz JD, Sheu TJ, et al. Elevated Lifetime Lead Exposure Impedes Osteoclast Activity and Produces an Increase in Bone Mass in Adolescent Mice. Toxicol Sci 2016;149:277-88.

29. Carmouche JJ, Puzas JE, Zhang X, et al. Lead Exposure Inhibits Fracture Healing and Is Associated with Increased Chondrogenesis, Delay in Cartilage Mineralization, and a Decrease in Osteoprogenitor Frequency. Environ Health Perspect 2005;113:749-55.

30. Abbas S, Khan K, Khan MP, et al. Developmental exposure to $\mathrm{As}, \mathrm{Cd}$, and $\mathrm{Pb}$ mixture diminishes skeletal growth and causes osteopenia at maturity via osteoblast and chondrocyte malfunctioning in female rats. Toxicol Sci 2013;134:207-20.

Cite this article as: Chang L, Shen S, Zhang Z, Song X, Jiang Q. Study on the relationship between age and the concentrations of heavy metal elements in human bone. Ann Transl Med 2018;6(16):320. doi: 10.21037/atm.2018.08.09 